\title{
Recent Developments in Option Pricing
}

\author{
Hui Gong ${ }^{1}$, Aerambamoorthy Thavaneswaran ${ }^{2}$, You Liang ${ }^{2}$ \\ ${ }^{1}$ Department of Mathematics \& Computer Science, Valparaiso University, Valparaiso, USA \\ ${ }^{2}$ Department of Statistics, University of Manitoba, Winnipeg, Canada \\ E-mail: hughgong@gmail.com, thava@temple.edu,umlian33@cc.umanitoba.ca \\ Received September 1, 2011; revised September 28, 2011; accepted October 10, 2011
}

\begin{abstract}
In this paper, we investigate recent developments in option pricing based on Black-Scholes processes, pure jump processes, jump diffusion process, and stochastic volatility processes. Results on Black-Scholes model with GARCH volatility (Gong, Thavaneswaran and Singh [1]) and Black-Scholes model with stochastic volatility (Gong, Thavaneswaran and Singh [2]) are studied. Also, recent results on option pricing for jump diffusion processes, partial differential equation (PDE) method together with FFT (fast Fourier transform) approximations of Pillay and O' Hara [3] and a recently proposed method based on moments of truncated lognormal distribution (Thavaneswaran and Singh [4]) are also discussed in some detail.
\end{abstract}

Keywords: Stochastic Volatility, Black-Scholes Partial Differential Equations, Option Pricing, Monte Carlo

\section{Introduction}

Option pricing is one of the major areas in modern financial theory and practice. Since Black, Scholes, and Merton introduced their path-breaking work on option pricing, there has been explosive growth in derivatives trading activities in the worldwide financial markets. The main contribution of the seminal work of Black and Scholes [5,6] and Merton [7] was the introduction of a preference-free option pricing formula that does not involve an investor's risk preferences and subjective views. Due to its compact form and computational simplicity, the Black-Scholes formula enjoys great popularity in the finance industries and is based on the strong assumption that the volatility of the stock returns is constant. However, implied volatility of the stock prices suggests stochastic volatility models are more appropriate to model the stock price. The most popular approach is to use the Heston model (Heston [8,9]), which assumes that the underlying asset follows the Black-Scholes model but the volatility is stochastic and follows the Cox Ingersoll Ross process (Cox, Ingersoll and Ross [10]). The empirical results of Bakshi, Cao and Chen [11] suggest that taking stochastic volatility into account is important in option pricing. Motivated by the theoretical considerations, Scott [12], Hull and White [13,14], Ritchken and Trevor [15], and Wiggins [16] generalized the Black-Scholes model to allow stochastic volatility.

Heston and Nandi [17] first provided a solid theoretical foundation based on the concept of locally risk-neutral valuation relationship for option valuation under nonlinear GARCH models using characteristic functions. Heston and Nandi [17], Elliot, Siu and Chan [18], Christoffersen, Heston and Jacobs [19], and Mercuri [20] among others derived closed form option pricing formula under various GARCH models. Recently, Badescu and Kulpeger [21], Barone-Adesi et al. [22,23], and Gong, Thavaneswaran and Singh [1] including others have studied option pricing under GARCH volatility. Thavaneswaran, Peiris and Singh [24] and Thavaneswaran, Singh and Appadoo [25] studied option pricing using the moment properties of the truncated lognormal distribution. Gong, Thavaneswaran and Singh [1,2] studied Black-Scholes models with GARCH volatility and with stochastic volatility as in Taylor [26]. They carried out extensive empirical analysis of the European call option valuation for S \& P 100 index and showed that the proposed method outperformed other compelling stochastic volatility pricing models. In Thavaneswaran and Singh [4], option pricing for jump diffusion process with stochastic volatility was studied by viewing option pricing as a truncated moment of a lognormal distribution. Pillay and O' Hara [3] studied the FFT based option pricing under a mean reverting process with stochastic volatility and jumps by using the PDE approach.

In this paper, we first derive the Black Scholes partial differential equation for stochastic volatility models and then obtain closed-form solutions for the resulting PDEs. The rest of this paper is organized as follows. In Section 
2, we study option pricing for pure jump processes, jump diffusion models, stochastic volatility models, and jump diffusion models with stochastic volatility. Moreover, closed form solutions are obtained by solving the two-dimensional partial differential equations for stock price in some examples. Section 3 closes the paper with conclusions.

\section{Option Pricing and Partial Differential Equations}

Consider the stock and bond model as in Steele [27]

$$
\mathrm{d} X(t)=\mu(t, X(t)) \mathrm{d} t+\sigma(t, X(t)) \mathrm{d} W(t),
$$

and

$$
\mathrm{d} \beta(t)=r(t, X(t)) \beta(t) \mathrm{d} t,
$$

where all of the model coefficients

$\mu(t, X(t)), \sigma(t, X(t))$, and $r(t, X(t))$ are given by explicit functions of the current time and current stock price. First, we will use the coefficient matching method to show that arbitrage price at time $t$ of a European option with terminal time $T$ and payout $h(t, X(t))$ is given by $f(t, X(t))$ where $f(t, x)$ is the solution of the partial differential equation (PDE)

$$
\begin{aligned}
& f_{t}(t, x)+r(t, x) x f_{x}(t, x)+\frac{1}{2} \sigma^{2}(t, x) f_{x x}(t, x) \\
= & r(t, x) f(t, x)
\end{aligned}
$$

with terminal condition

$$
f(T, x)=h(x) .
$$

If we let $a(t)$ denote the number of units of stock that we hold in the replicating portfolio at time $t$ and let $b(t)$ denote the corresponding number of units of the bond, then the total value of the portfolio at time $t$ is

$$
V(t)=a(t) X(t)+b(t) \beta(t) .
$$

The condition where the portfolio replicates the contingent claim at time $\mathrm{T}$ is simply

$$
V(T)=h(X(T)) .
$$

From the self-financing condition

$$
\mathrm{d} V(t)=a(t) \mathrm{d} X(t)+b(t) \mathrm{d} \beta(t)
$$

and the models for the stock and bond, we have

$$
\begin{aligned}
\mathrm{d} V(t)= & (a(t) \mu(t, X(t))+b(t) r(t, X(t)) \beta(t)) \mathrm{d} t \\
& +a(t) \sigma(t, X(t)) \mathrm{d} W(t) .
\end{aligned}
$$

Then from our assumption that $V(t)=h(t, X(t))$ for any twice differentiable function $f(t, x)$ and the Itô formula, we have (2.4).

When we equate the drift and diffusion coefficients from Equation (2.3) and Equation (2.4), we find a simple expression for the size of the stock portion of our replicating portfolio:

$$
a(t)=f_{x}(t, X(t)),
$$

and find

$$
\begin{aligned}
& \mu(t, X(t)) f_{x}(t, X(t))+b(t) r(t, X(t)) \beta(t) \\
= & f_{t}(t, X(t))+\frac{1}{2} f_{x x}(t, X(t)) \sigma^{2}(t, X(t)) \\
+ & f_{x}(t, X(t)) \mu(t, X(t)) .
\end{aligned}
$$

The $\mu(t, X(t)) f_{x}(t, X(t))$ terms cancel, and $b(t)$ is given by

$b(t)=$

$\frac{1}{r(t, X(t)) \beta(t)}\left(f_{t}(t, X(t))+\frac{1}{2} f_{x x}(t, X(t)) \sigma^{2}(t, X(t))\right)$.

Because $V(t)$ is equal to both $f(t, X(t))$ and $a(t) X(t)+b(t) \beta(t)$, the values for $a(t)$ and $b(t)$ give us a PDE for $f(t, X(t))$ :

$$
\begin{aligned}
f(t, X(t))= & f_{x}(t, X(t)) X(t)+\frac{1}{r(t, X(t))} \\
& \times\left(f_{t}(t, X(t))+\frac{1}{2} f_{x x}(t, X(t)) \sigma^{2}(t, X(t))\right) .
\end{aligned}
$$

Now, when we replace $X(t)$ by $x$, we arrive at the general Black-Scholes PDE (2.1) and its terminal boundary condition (2.2).

We can solve the Black-Scholes PDE to get the time- $t$ call price. The link between the stochastic solution to PDE and the martingale approcach is given by the following Feynman-Kac Theorem.

Theorem 2.1 A function $f(t, x)$ defined by

$$
f(t, x)=\mathbb{E}\left[e^{-\int_{t}^{T} V(X(\tau)) \mathrm{d} \tau} h(X(T)) \mid X(t)=x\right],
$$

where $\mathrm{d} X(t)=\mu(t, X(t)) \mathrm{d} t+\sigma(t, X(t)) \mathrm{d} W(t)$ satisfies partial differential equation

$$
\begin{aligned}
\mathrm{d} V(t) & =f_{t}(t, X(t)) \mathrm{d} t+\frac{1}{2} f_{x x}(t, X(t)) \sigma^{2}(t, X(t)) \mathrm{d} t+f_{x}(t, X(t)) \mathrm{d} X(t) \\
& =\left(f_{t}(t, X(t))+\frac{1}{2} f_{x x}(t, X(t)) \sigma^{2}(t, X(t))+f_{x}(t, X(t)) \mu(t, X(t))\right) \mathrm{d} t+f_{x}(t, X(t)) \sigma(t, X(t)) \mathrm{d} W(t)
\end{aligned}
$$




$$
\begin{aligned}
& f_{t}(t, x)+\mu(t, x) f_{x}(t, x)+\frac{1}{2} \sigma^{2}(t, x) f_{x x}(t, x) \\
= & V(t, x) f(t, x)
\end{aligned}
$$

with the terminal condition $f(T, x)=h(x)$.

Consider the Black-Scholes PDE

$$
f_{t}(t, x)+r x f_{x}(t, x)+\frac{1}{2} \sigma^{2} x^{2} f_{x x}(t, x)=r f(t, x),
$$

with the terminal condition: (a) $f(T, x)=(x-K)^{+}$; (b) $f(T, x)=h(x)$ for some function $h:(0, \infty) \rightarrow \mathbb{R}$; (c) $f(T, x)=x^{v} ;(\mathrm{d}) \quad f(T, x)=\log (x)$.

The above the Black-Scholes equation has a stochastic solution of the form

$$
f(t, x)=\mathbb{E}_{\mathbb{Q}}\left[\mathrm{e}^{-r(T-t)} f(X(T)) \mid X(t)=x\right],
$$

where, under the neutral risk measure $\mathbb{Q}$,

$$
\mathrm{d} X(t)=r X(t) \mathrm{d} t+\sigma X(t) \mathrm{d} W(t),
$$

and $X(t)$ is a geometric Brownian motion of the form

$$
X(T)=X(t) \exp \left\{\left(r-\sigma^{2} / 2\right)(T-t)+\sigma W(T-t)\right\} .
$$

For specific terminal conditions, the closed form time$t$ price can be obtained by finding the stochastic solutions. In Case (a), the time- $t$ price is given by the Black-Scholes formula

$$
\begin{aligned}
& f(t, x) \\
= & \mathbb{E}_{Q}\left[\mathrm{e}^{-r(T-t)}(X(T)-K)^{+} \mid X(t)=x\right] \\
= & \mathrm{e}^{-r(T-t)} \frac{1}{\sqrt{2 \pi}} \int_{-\infty}^{\infty}\left(\mathrm{e}^{\log (x)+\left(r-\sigma^{2} / 2\right)(T-t)+\sigma \sqrt{T-t z}}-K\right)^{+} \mathrm{e}^{-z^{2} / 2} \mathrm{~d} z \\
= & x \Phi\left(\mathrm{d}_{1}\right)-\mathrm{e}^{-r(T-t)} K \Phi\left(\mathrm{d}_{2}\right),
\end{aligned}
$$

where

$$
\mathrm{d}_{1}=\frac{\log (x / K)+\left(r+\sigma^{2} / 2\right)(T-t)}{\sigma \sqrt{T-t}}, \mathrm{~d}_{2}=\mathrm{d}_{1}-\sigma \sqrt{T-t} .
$$

In Case (b), the time-t price can be represented as

$$
\begin{aligned}
& f(t, x) \\
= & \mathbb{E}_{Q}\left[\mathrm{e}^{-r(T-t)} f\left(X(t) \mathrm{e}^{\left(\mu-\sigma^{2} / 2\right)(T-t)+\sigma W(T-t)}\right) \mid X(t)=x\right] \\
= & \mathrm{e}^{-r(T-t)} \mathbb{E}\left[f\left(x \mathrm{e}^{\left(r-\sigma^{2} / 2\right)(T-t)+\sigma \sqrt{T-t} Z}\right)\right] .
\end{aligned}
$$

In Case (c), for some real $v$,

$$
\begin{aligned}
& f(t, x) \\
= & \mathbb{E}_{Q}\left[\mathrm{e}^{-r(T-t)}(X(t))^{v} \mathrm{e}^{v\left(\mu-\sigma^{2} / 2(T-t)+v \sigma W(T-t)\right.} \mid X(t)=X\right] \\
= & \mathrm{e}^{-r(T-t)} X^{v} \mathbb{E}\left[\mathrm{e}^{v\left(r-\sigma^{2} / 2\right)(T-t)+v \sigma W(T-t)}\right] \\
= & x^{v} \mathrm{e}^{-(1-v)\left(r+\sigma^{2} / 2\right)(T-t)} .
\end{aligned}
$$

In Case (d),

$$
\begin{aligned}
& f(t, x) \\
= & \mathbb{E}_{Q}\left[\mathrm{e}^{-r(T-t)} \log \left(X(t) \mathrm{e}^{\left(\mu-\sigma^{2} / 2\right)(T-t)+\sigma W(T-t)}\right) \mid X(t)=x\right] \\
= & \mathrm{e}^{-r(T-t)}\left(\log (x)+\left(\mu-\sigma^{2} / 2\right)(T-t)\right) .
\end{aligned}
$$

\subsection{Option Pricing for Pure Jump Processes (PDE Approach)}

In order to price European options based on jump processes (Vecer [28]), we need to know the evolution of both $X_{Y}(t)$ and $Y_{X}(t)$ in order to determine both martingale measures $\mathbb{P}^{X}$ and $\mathbb{P}^{Y}$. It is possible to preserve the symmetry of the evolution of the prices with the exception that the jump preserves the direction: when $X_{Y}(t)$ jumps up, $Y_{X}(t)$ jumps down, and vise versa. The jump $N(t)$ belongs to the pair of and $Y$; it cannot be individualized to one asset in contrast to the geometric Brownian motion model, where the noise factor $W^{X}$ is associated with the asset $X$, and the noise factor $W^{Y}$ is associated with the asset $Y$. In the case of Poisson evolution, it is the intensity $\lambda$ of the Poisson process that is associated with the particular asset. Under the $\mathbb{P}^{X}$ measure, $N(t)$ has intensity $\lambda^{X} t$ and the process $N(t)-\lambda^{X} t$ is a martingale, while under the $\mathbb{P}^{Y}$ measure, $N(t)$ has intensity $\lambda^{Y} t$ and the process $N(t)-\lambda^{Y} t$ is a martingale.

The price process $X_{Y}(t)$ driven by a SDE of the form

$$
d X_{Y}(t)=\left(\mathrm{e}^{\gamma}-1\right) X_{Y}(t-) d\left(N(t)-\lambda^{Y} t\right),
$$

has a solution as a geometric Poisson process

$$
X_{Y}(t)=X_{Y}(0) \exp \left(\gamma N(t)-\left(\mathrm{e}^{\gamma}-1\right) \lambda^{Y} t\right) .
$$

The inverse price process $Y_{X}(t)$ satisfy the SDE of the form

$$
\mathrm{d} Y_{X}(t)=\left(\mathrm{e}^{-\gamma}-1\right) Y_{X}(t-) \mathrm{d}\left(N(t)-\mathrm{e}^{\gamma} \lambda^{X} t\right),
$$

and has a geometric Poisson process representation

$$
Y_{X}(t)=Y_{X}(0) \exp \left(-\gamma N(t)-\left(\mathrm{e}^{-\gamma}-1\right) \lambda^{X} t\right) .
$$

The values of $\lambda^{Y}$ and $\lambda^{X}$ are linked by the relationship $\lambda^{X}=e^{\gamma} \lambda^{Y}$, where $\gamma$ is the size of the jump of $\log X_{Y}(t)$. Let $V$ be a contract that pays off $f^{Y}\left(X_{Y}(T)\right)$ units of an asset $Y$ at time $T$. The price of the contract $V$ with respect to the reference $Y$ is given by

$$
V_{Y}(t)=\mathbb{E}_{t}^{Y}\left[f^{Y}\left(X_{Y}(T)\right)\right] .
$$

The conditional expectation on the right hand side of the above equation with respect to $Y$ is a $\mathbb{P}^{Y}$ martingale, and its value depends only on the price of $X_{Y}(t)$. Thus 
we can write

$$
u^{Y}(t, x)=\mathbb{E}_{t}^{Y}\left[f^{Y}\left(X_{Y}(T)\right) \mid X_{Y}(t)=x\right] .
$$

Similarly we can also compute the price of this contract with respect to a reference asset $X$ as

$$
V_{X}(t)=\mathbb{E}_{t}^{X}\left(f^{X}\left(Y_{X}(T)\right)\right),
$$

where $f^{X}$ is a payoff function in terms of the asset $X$. And the price function $u^{X}(t, x)$ is defined as

$$
u^{X}(t, x)=\mathbb{E}_{t}^{X}\left[f^{Y}\left(Y_{X}(T)\right) \mid Y_{X}(t)=x\right] .
$$

Then the following theorem (Vecer [28]) gives the form of the PDEs for $u^{X}(t, x)$ and $u^{Y}(t, x)$.

Theorem 2.2 (a) The price function based on the geometric Poisson process (2.5),

satisfies

$$
u^{X}(t, x)=\mathbb{E}_{t}^{X}\left[f^{X}\left(Y_{X}(T)\right) \mid Y_{X}(t)=x\right]
$$

$$
\begin{aligned}
& u_{t}^{X}(t, x) \\
& +\lambda^{X}\left(u_{t}^{X}\left(t, \mathrm{e}^{\gamma} x\right)-u^{X}(t, x)-\left(\mathrm{e}^{-\gamma}-1\right) x u_{x}^{X}(t, x)\right)=0
\end{aligned}
$$

with the terminal condition

$$
u^{X}(T, x)=f^{X}(x) .
$$

(b) The price function based on the geometric Poisson process (2.6), $u^{Y}(t, x)=\mathbb{E}_{t}^{Y}\left[f^{Y}\left(X_{Y}(T)\right) \mid X_{Y}(t)=x\right]$ satisfies $u_{t}^{Y}(t, x)+\lambda^{Y}\left(u_{t}^{Y}\left(t, \mathrm{e}^{\gamma} x\right)-u^{Y}(t, x)-\left(\mathrm{e}^{\gamma}-1\right) x u_{x}^{Y}(t, x)\right)=0$

with the terminal condition

$$
u^{Y}(T, x)=f^{Y}(x) .
$$

Proof of this theorem is given in [28] (pp. 249-250).

For a geometric Poisson model (Vecer [28]) for two no-arbitrage assets $X$ and $Y$, where the price follows

$$
\mathrm{d} X_{Y}(t)=\left(\mathrm{e}^{\gamma}-1\right) X_{Y}(t-) \mathrm{d}\left(N(t)-\lambda^{Y} t\right),
$$

and by letting $N(t)=m \leq k$, the price of a contract that pays off $V_{T}=I(N(T)=k) Y_{T}$ in terms of a reference asset $Y$ is given by

$$
\begin{aligned}
V_{Y}(t) & =\mathbb{E}_{t}^{Y}\left[V_{Y}(T)\right]=\mathbb{E}_{t}^{Y}[\mathbb{I}(N(T)=k)] \\
& =\mathbb{P}_{t}^{Y}(N(T)=k)=\mathbb{P}_{t}^{Y}(N(T)-N(t)=k-m) \\
& =\frac{e^{-\lambda^{Y}(T-t)}\left(\lambda^{Y}(T-t)\right)^{k-m}}{(k-m) !} .
\end{aligned}
$$

Moreover, the price of $V_{X}(t)$ using the reference asset $X$ is given by

$$
V_{X}(t)=V_{Y}(t) Y_{X}(t)=Y_{X}(t) \frac{\mathrm{e}^{-\lambda^{Y}(T-t)}\left(\lambda^{Y}(T-t)\right)^{k-m}}{(k-m) !} .
$$

\subsection{Option Pricing for Jump Diffusion Processes}

Recently Pillay and O’ Hara [3] have studied the FFT based option pricing under a mean reverting process with stochastic volatility and jumps by finding a closed form of expression for a conditional characteristic function of the log asset process and then apply the FFT method to compute the option price.

Let $(\Omega, \mathcal{F}, \mathbb{Q})$ be a probability space on which are defined two Brownian motion processes. Let $\mathcal{F}_{t}$ be the filtration generated by these Brownian motions. Suppose that $\mathbb{Q}$ is a risk neutral probability under which the asset price process $S(t)$ and volatility process $v(t)$ are governed by the following dynamics:

$$
\begin{aligned}
& \mathrm{d} S(t)=k(\theta(t)-\ln S(t)) S(t) \mathrm{d} t+\sqrt{v(t)} \mathrm{d} W_{1}(t), \\
& \mathrm{d} v(t)=b(a(t)-v(t)) \mathrm{d} t+\sigma \sqrt{v(t)} \mathrm{d} W_{2}(t), \\
& \mathrm{d} W_{1}(t) \mathrm{d} W_{2}(t)=\rho \mathrm{d} t,
\end{aligned}
$$

where $\theta(t)$ is a deterministic function that represents the equilibrium mean level of the asset against time, $k$ is the mean reverting intensity of the asset, $a(t)$ is a deterministic function that describes the equilibrium mean level of the volatility process against time and $b$ is the mean reversion speed of the volatility process. The constant $\sigma$ is the volatility coefficient of the volatility process, and $W_{1}(t)$ and $W_{2}(t)$ are correlated with correlation coefficient $\rho$.

On the probability space $(\Omega, \mathcal{F}, \mathbb{Q})$, a Poisson process $N(t)$ is further defined for all $0 \leq t \leq T$, with a constant intensity parameter $\lambda>0$. The process $N(t)$ is assumed to be independent of both $W_{1}(t)$ and $W_{2}(t)$. Furthermore, a sequence of random variables $e^{J_{i}}$ for $1 \leq i \leq N(t)$ is defined to represent the jump sizes of the Poisson process. Each of the $e^{J_{i}}$ 's are log-normally, identically and independently distributed over time, where $J_{i} \sim N\left(\mu, \gamma^{2}\right)$ and $\gamma>0$. Then by defining the following process $X(t)=\ln S(t)$ and applying the Itô-Doebin formula to the two-factor mean reverting process with stochastic volatility and jumps we have

$$
\begin{aligned}
& \mathrm{d} X(t)=k\left(\theta-\frac{v(t)}{2 k}-\frac{\lambda m}{k}-X(t)\right) \mathrm{d} t+\sqrt{v(t)} \mathrm{d} W_{1}(t) \\
&+\left(\mathrm{e}^{J}-1\right) X(t-) \mathrm{d} N(t) \\
& \mathrm{d} v(t)= b(a-v(t)) \mathrm{d} t+\sigma \sqrt{v(t)} \mathrm{d} W_{2}(t) \\
& \mathrm{d} W_{1}(t) \mathrm{d} W_{2}(t)=\rho \mathrm{d} t
\end{aligned}
$$

The conditional characteristic function of the $X(t)$ process (2.7) is defined as 


$$
\phi_{t}(u) \equiv \mathrm{E}_{\mathbb{Q}}\left[\mathrm{e}^{i u X(T)} \mid \mathcal{F}_{t}\right] .
$$

The method of Wong and Lo [29] has applied to compute the characteristic function of the process (2.7).

Duffie, Pan and Singleton [30] introduced a generalized Feynman-Kac theorem for affine jump diffusion processes. By defining the following function:

$$
\begin{aligned}
f(u ; t, x, v) & =\mathrm{E}_{\mathbb{Q}}\left[\mathrm{e}^{i u X(T)} \mid X(t)=x, v(t)=v\right] \\
& =\mathrm{e}^{-r T} \mathrm{E}_{\mathbb{Q}}\left[\mathrm{e}^{r T} \mathrm{e}^{i u X(T)} \mid X(t)=x, v(t)=v\right],
\end{aligned}
$$

which can be viewed as a contingent claim that pays $e^{r T+i u x}$ at time $T$, where $r$ is a constant interest rate, $X(t)$ is the mean reverting asset price process with jumps defined by (2.7) and $v(t)$ is the volatility process specified by (2.8), the generalized Feynman-Kac theorem implies that $f(u ; t, x, v)$ solves the following partial integro-differential equation (PIDE):

$$
\begin{aligned}
& f_{t}+k\left(\theta-\frac{v}{2 k}-\frac{\lambda m}{k}-x\right) f_{x}+\frac{1}{2} v f_{x x}+b(a-v) f_{v}+\frac{1}{2} \sigma^{2} v f_{v v} \\
& +\rho \sigma v f_{x v}+\lambda \int_{-\infty}^{\infty}(f(u ; t, x+J, v)-f(u ; t, x, v)) q(J) \mathrm{d} J=0,
\end{aligned}
$$

where $q(J)$ is the distribution function of the random variable $J$ and $\lambda>0$ is the constant intensity parameter of the Poisson process $N(t)$.

The coefficients, $k(\theta-v / 2 k-\lambda m / k-x)$ and $\sqrt{v(t)}$, of the mean reverting asset price process (2.7) and the coefficients, $b(a-v(t))$ and $\sigma \sqrt{v(t)}$ of the volatility process (2.8) are all affine in nature. It follows that the function $f(u ; t, x, v)$ is of exponential affine form, and hence the solution of (2.11) has the form

$$
f(u ; t, x, v)=\exp \{B(t, T)+C(t, T) x+D(t, T) v+\text { iux }\},
$$

where $B(t, T), C(t, T)$ and $D(t, T)$ are deterministic functions of $t$. From (2.10), it is clear that

$$
f(u ; T, x, v)=\exp \{\text { iux }\},
$$

which is the terminal condition of PIDE (2.11). This implies that

$$
B(T, T)=0, C(T, T)=0, D(T, T)=0 .
$$

Solving the PIDE (2.11) with the terminal conditions (2.12), the conditional characteristic function of the mean reverting process (2.7) with stochastic volatility (2.8) is

$$
\phi_{t}(u)=\exp \{B(t, T)+C(t, T) x+D(t, T) v+\operatorname{iux}\}
$$

where $B(t, T), C(t, T)$ and $D(t, T)$ are given in (2.13).

The detail proof of the results is given in Pillay and $\mathrm{O}^{\prime}$ Hara [3].

\subsection{Option Pricing for Stochastic Volatility Models}

Given a twice-differentiable continuous function $\{f(\phi ; t, x, v)\}_{t \leq T}$, the price process $X(t)$ and the volatility Process $v(t)$ follow the following stochastic volatility processes

$$
\begin{aligned}
& \mathrm{d} X(t)=\mu(x) \mathrm{d} t+\sigma(x) \mathrm{d} W_{1}(t), \\
& \mathrm{d} v(t)=\mu(v) \mathrm{d} t+\sigma(v) \mathrm{d} W_{2}(t), \\
& X(t)=\ln S(t),
\end{aligned}
$$

where $\mathrm{d} W_{1}(t) \mathrm{d} W_{2}(t)=\rho \mathrm{d} t$. Then the PDE of $f$ can be obtained by using Itô formula, see (2.14).

Setting the drift term to zero we have

$$
\begin{aligned}
& f_{\tau}+\mu(x) f_{x}+\mu(x) f_{v}+\rho \sigma(x) \sigma(v) f_{x v} \\
& +\frac{1}{2} \sigma^{2}(x) f_{x x}+\frac{1}{2} \sigma^{2}(v) f_{v v}=0 .
\end{aligned}
$$

$$
\begin{aligned}
& B(t, T)=\left(\frac{\lambda m}{k}-\theta\right) i u\left(\mathrm{e}^{-k(T-t)}-1\right)-a b \int_{t}^{T} D(s, T) \mathrm{d} s+\Lambda(u)(T-t), C(t, T)=i u\left(\mathrm{e}^{-k(T-t)}-1\right), \\
& D(t, T)=U e^{-k(T-t)}+\frac{e^{-b(T-t)} V e^{-k(T-t)}}{\frac{-1}{U(1)}+\frac{\sigma^{2}}{2 k} \int_{1}^{e^{-k(T-t)}}}, U(\tau)=\frac{2 k \tau}{\tau^{2}-1} \frac{\left(\sqrt{1-\rho^{2}}-\rho i\right) \frac{\sigma u}{2 k} \Phi\left(a^{*}, b^{*}, \frac{\tau}{\zeta}\right)+\frac{a^{*}}{b^{*} \zeta} \Phi\left(a^{*}+1, b^{*}+1, \frac{\tau}{\zeta}\right)}{\Phi\left(a^{*}, b^{*}, \frac{\tau}{\zeta}\right)}, \\
& V(\tau)=\frac{\Phi\left(a^{*}, b^{*}, \frac{1}{\zeta}\right)}{\Phi\left(a^{*}, b^{*}, \frac{\tau}{\zeta}\right)} e^{\frac{\sigma u}{k}(1-\tau) \sqrt{1-\rho^{2}}}, a^{*}=\frac{\left(\sqrt{1-\rho^{2}}+\rho\right) \frac{b^{*}}{2}+\frac{\sigma}{4 k}}{\sqrt{1-\rho^{2}}}, b^{*}=1-\frac{b}{k}, \zeta=\frac{-k}{\sigma u \sqrt{1-\rho^{2}}}, \Lambda(u)=\lambda\left(\mathrm{e}^{i u \mu-\frac{1}{2} u^{2} \gamma^{2}}-1\right) .
\end{aligned}
$$

$$
\mathrm{d} f=\left(f_{\tau}+\mu(x) f_{x}+\mu(v) f_{v}+\rho \sigma(x) \sigma(v) f_{x v}+\frac{1}{2} \sigma(x)^{2} f_{x x}+\frac{1}{2} \sigma(v)^{2} f_{v v}\right) \mathrm{d} t+\sigma(x) f_{x} \mathrm{~d} W_{1}(t)+\sigma(v) f_{v} d W_{2}(t) .
$$


One can calculate the asset price by inverting its conditional characteristic function $\mathbb{E}\left(S(T)^{i \phi} \mid \mathcal{F}\right)_{t}$. The conditional characteristic function of the $X(t)$ process is defined as

$$
\phi_{t}(u) \equiv \mathrm{E}_{\mathbb{Q}}\left[\mathrm{e}^{i \phi X(T)} \mid \mathcal{F}_{t}\right] .
$$

Furthermore, if one defining the following function

$$
f(\phi ; t, x, v)=\mathrm{E}_{\mathbb{Q}}\left[\mathrm{e}^{i \phi X(T)} \mid X(t)=x, v(t)=v\right],
$$

then solution of (2.15) is the characteristic function. To solve for the characteristic function explicitly, consider an exponential affine form

$$
f(\phi ; t, x, v)=\exp \{B(\tau)+C(\tau) x+D(\tau) v+i \phi x\}
$$

where $\tau=T-t$ and $B(\tau=0)=C(\tau=0)=D(\tau=0)=0$. Take derivatives of (2.16) with respect to $x, v$ and $\tau$, we get

$$
\begin{aligned}
f_{x} & =(C(\tau)+i \phi) f(\phi ; t, x, v) \\
f_{v} & =D(\tau) f(\phi ; t, x, v) \\
f_{x x} & =(C(\tau)+i \phi)^{2} f(\phi ; t, x, v) \\
f_{v v} & =D^{2}(\tau) f(\phi ; t, x, v) \\
f_{x v} & =(C(\tau)+i \phi) D(\tau) f(\phi ; t, x, v) \\
f_{\tau} & =-\left(B_{\tau}(\tau)+C_{\tau}(\tau) x+D_{\tau}(\tau) v\right) f(\phi ; t, x, v) .
\end{aligned}
$$

Substituting (2.17) into (2.15), it yields

$$
\begin{aligned}
& -\left(B_{\tau}(\tau)+C_{\tau}(\tau) x+D_{\tau}(\tau) v\right)+\mu(x)(C(\tau)+i \phi) \\
& +\mu(v) D(\tau)+\rho \sigma(x) \sigma(v)(C(\tau)+i \phi) D(\tau) \\
& +\frac{1}{2} \sigma^{2}(x)(C(\tau)+i \phi)^{2}+\frac{1}{2} \sigma^{2}(v) D^{2}(\tau)=0 .
\end{aligned}
$$

Equation (2.18) leads to a system of ODEs. We can get the characteristic function by solving this ODE system.

As an example, for the Stochastic Volatility model studied by Christoffersen, Heston and Jacobs [19] defined by

$$
\begin{aligned}
& \mathrm{d} X(t)=(R+u v(t)) \mathrm{d} t+\sqrt{v(t)} \mathrm{d} W_{1}(t), \\
& \mathrm{d} v(t)=(a-c v(t)) \mathrm{d} t+\xi \sqrt{v(t)} \mathrm{d} W_{2}(t), \\
& X(t)=\ln S(t),
\end{aligned}
$$

where $\mathrm{d} W_{1}(t) \mathrm{d} W_{2}(t)=\rho \mathrm{d} t$, and we know the equations that $\mu(x)=R+u v(t), \quad \sigma(x)=\sqrt{v(t)}, \quad \mu(v)=a-c v(t)$ and $\sigma(v)=\xi \sqrt{v(t)}$. Substitute them into (2.16), we have

$$
\begin{aligned}
0= & v(t)(u(C(\tau)+i \phi)-c D(\tau)+\rho \xi(C(\tau)+i \phi) D(\tau) \\
& \left.+\frac{1}{2}(C(\tau)+i \phi)^{2}+\frac{1}{2} \xi^{2} D^{2}(\tau)-D_{\tau}(\tau)\right)-C_{\tau}(\tau) x(t) \\
& +R(C(\tau)+i \phi)+a D(\tau)-B_{\tau}(\tau),
\end{aligned}
$$

and the system of ODEs becomes

$$
\begin{gathered}
0=u(C(\tau)+i \phi)-c D(\tau)+\rho \xi(C(\tau)+i \phi) D(\tau) \\
+\frac{1}{2}(C(\tau)+i \phi)^{2}+\frac{1}{2} \xi^{2} D^{2}(\tau)-D_{\tau}(\tau), \\
0=-C_{\tau}(\tau), \\
0=R(C(\tau)+i \phi)+a D(\tau)-B_{\tau}(\tau) .
\end{gathered}
$$

It is clear from (2.20) and $C(\tau=0)=0$ that $C(\tau)=0$. Hence, (2.19) and (2.21) turn out to be

$$
\begin{aligned}
& D_{\tau}(\tau)=u i \phi-c D(\tau)+\rho \xi i \phi D(\tau)-\frac{1}{2} \phi^{2}+\frac{1}{2} \xi^{2} D^{2}(\tau), \\
& B_{\tau}(\tau)=\operatorname{Ri} \phi+a D(\tau) .
\end{aligned}
$$

One can solve these two ODEs under the conditions $B(0)=0$ and $D(0)=0$ to obtain

$$
\begin{aligned}
& B(\tau)=R i \phi \tau+\frac{a}{\xi^{2}}\left(-(\rho \xi i \phi-c-\mathrm{d}) \tau-2 \ln \left(\frac{1-g e^{\mathrm{d} \tau}}{1-g}\right)\right), \\
& D(\tau)=\frac{\rho \xi i \phi-c-\mathrm{d}}{\xi^{2}}\left(\frac{e^{\mathrm{d} \tau}-1}{1-g e^{\mathrm{d} \tau}}\right),
\end{aligned}
$$

where

$$
g=\frac{\rho \xi i \phi-c-\mathrm{d}}{\rho \xi i \phi-c+\mathrm{d}}, \mathrm{d}=\sqrt{(\rho \xi i \phi-c)^{2}-\xi^{2}\left(2 u i \phi-\phi^{2}\right)} .
$$

The FFT method can be used to obtain the call price from the conditional characteristic function.

\subsection{Option Pricing for Jump Diffusion Model with Stochastic Volatility}

Thavaneswaran and Singh [4] considered the price process $S(t)$ following a conditional jump diffusion model

$$
\begin{aligned}
& \mathrm{d} \log S(t)=v \mathrm{~d} t+\theta(t) \mathrm{d} W(t)+Y \mathrm{~d} N(t), \\
& y(t)=\log \frac{S(t)}{S(t-1)}-\mathbb{E}\left[\log \frac{S(t)}{S(t-1)}\right]=\theta(t) Z(t),
\end{aligned}
$$

where $v \equiv r-\lambda m-\theta(t)^{2} / 2, m=\mathbb{E}\left[e^{Y}-1\right]$, and $W(t)$ is a standard Brownian motion, $\theta(t)$ is a stationary process, and the number of jumps $N(t)$ on $(0, t]$ follows a Poisson process with rate $\lambda$. Equivalently the model can be written as 


$$
\begin{aligned}
& \log \frac{S(t)}{S}=v t+\theta(t) W(t)+\sum_{i=1}^{N(t)} Y_{i}, \\
& y(t)=\log \frac{S(t)}{S(t-1)}-\mathbb{E}\left[\log \frac{S(t)}{S(t-1)}\right]=\theta(t) Z(t),
\end{aligned}
$$

where $Y_{i}$ 's are identically distributed independent normal random variables having mean $\mu$ and variance $\sigma^{2}$. Let $\Theta(t)^{2}=\theta(t)^{2}+n \sigma^{2} / T$. Then conditioning on $\Theta(t)$ and taking the expectation as in Theorem 1 in [4], the call price is given by

$$
\begin{aligned}
& C(S, T) \\
= & \sum_{n=1}^{\infty} \mathrm{e}^{-\lambda(1+m) T} \frac{(\lambda(1+m) T)^{n}}{n !} \mathrm{e}^{-r_{n} T} \mathbb{E}_{\Theta(t)}\left[\mathbb{E}\left[(S(T)-K)^{+}\right]\right] \\
= & \sum_{n=1}^{\infty} e^{-\lambda(1+m) T} \frac{(\lambda(1+m) T)^{n}}{n !} S \mathbb{E}_{\Theta(t)}[f(\Theta(t))]
\end{aligned}
$$

$$
-\sum_{n=1}^{\infty} e^{-\lambda(1+m) T} \frac{(\lambda(1+m) T)^{n}}{n !} K e^{-r_{n} T} \mathbb{E}_{\Theta(t)}[g(\Theta(t))]
$$

where

$$
\begin{gathered}
f(\Theta(t))=\Phi\left(\frac{\log \left(\frac{S}{K}\right)+r_{n} T+\frac{1}{2} \Theta(t)^{2}}{\Theta(t)}\right), \\
g(\Theta(t))=\Phi\left(\frac{\log \left(\frac{S}{K}\right)+r_{n} T+\frac{1}{2} \Theta(t)^{2}}{\Theta v}-\Theta(t),\right. \\
(S(T)-K)^{+}=\max \{S(T)-K, 0\} .
\end{gathered}
$$

$S$ is the initial value of $S(t), r$ is the risk-free interest rate, $K$ is the strike price, and $T$ is the expiry date. For given $N(t)=n$, we have $r_{n}=r-\lambda m+n \log (1+m) / T$ and the $\Theta(t)$ process has mean $\mu_{\Theta}$, variance $\sigma_{\Theta}^{2}$, skewness $\gamma^{\Theta}$, and kurtosis $\kappa^{(\Theta)}$.Then the following theorem is given in Thavaneswaran and Singh [4].

Theorem 2.3 For any twice continuously differentiable function $f(x)$ and $g(x)$, the call price is given by

$$
\begin{aligned}
& C(S, T) \\
= & \sum_{n=1}^{\infty} \mathrm{e}^{-\lambda(1+m) T} \frac{(\lambda(1+m) T)^{n}}{n !} S \mathbb{E}_{\Theta(t)}[f(\Theta(t))]-\sum_{n=1}^{\infty} \mathrm{e}^{-\lambda(1+m) T} \frac{(\lambda(1+m) T)^{n}}{n !} K^{-r_{n} T} \mathbb{E}_{\Theta(t)}[g(\Theta(t))] \\
= & \sum_{n=1}^{\infty} \mathrm{e}^{-\lambda(1+m) T} \frac{(\lambda(1+m) T)^{n}}{n !} S \times\left(f\left(\mathbb{E}\left[\Theta^{2}(t)\right]\right)+\frac{1}{2} f^{\prime \prime}\left(\mathbb{E}\left[\Theta^{2}(t)\right]\right)\left(\mathbb{E}\left[\Theta^{2}(t)\right]+\mathbb{E}^{2}[\Theta(t)]\right)^{2}\left(\kappa^{(\Theta)}+4 \mathbb{E}^{2}[\Theta(t)]-1\right)\right) \\
& -\sum_{n=1}^{\infty} \mathrm{e}^{-\lambda(1+m) T} \frac{(\lambda(1+m) T)^{n}}{n !} K^{-r_{n} T} \times\left(g\left(\mathbb{E}\left[\theta^{2}(t)\right]\right)+\frac{1}{2} g^{\prime \prime}\left(\mathbb{E}\left[\Theta^{2}(t)\right]\right)\left(E\left[\Theta^{2}(t)\right]+\mathbb{E}^{2}[\Theta(t)]\right)^{2}\left(\kappa^{(\Theta)}+4 \mathbb{E}^{2}[\Theta(t)]-1\right)\right) \\
= & \sum_{n=1}^{\infty} \mathrm{e}^{-\lambda(1+m) T} \frac{(\lambda(1+m) T)^{n}}{n !} S\left(f\left(\mathbb{E}\left[\Theta^{2}(t)\right]\right)+\frac{1}{2} f^{\prime \prime}\left(\mathbb{E}\left[\Theta^{2}(t)\right]\right)\left(\frac{1}{3} \kappa^{(y)}-1\right) \mathbb{E}^{2}\left[\Theta^{2}(t)\right]\right) \\
& -\sum_{n=1}^{\infty} \mathrm{e}^{-\lambda(1+m) T} \frac{(\lambda(1+m) T)^{n}}{n !} K \mathrm{e}^{-r_{n} T}\left(g\left(\mathbb{E}\left[\Theta^{2}(t)\right]\right)+\frac{1}{2} g^{\prime \prime}\left(\mathbb{E}\left[\Theta^{2}(t)\right]\right)\left(\frac{1}{3} \kappa^{(y)}-1\right) \mathbb{E}^{2}\left[\Theta^{2}(t)\right]\right)
\end{aligned}
$$

where

$$
\kappa^{(\Theta)}=\frac{\mathbb{E}\left[(\Theta(t)-\mathbb{E}[(\Theta(t))])^{4}\right]}{\mathbb{E}^{2}\left[(\Theta(t)-\mathbb{E}[\Theta(t)])^{2}\right]}
$$

$$
f\left(\mathbb{E}\left[\Theta^{2}(t)\right]\right)=\Phi\left(\frac{\log \left(\frac{S}{K}\right)+r_{n} T+\frac{1}{2} \mathbb{E}\left[\Theta^{2}(t)\right]}{\sqrt{\mathbb{E}\left[\Theta^{2}(t)\right]}}\right),
$$

is the kurtosis of volatility process $\Theta(t)$,

$$
\kappa^{(y)}=\frac{\mathbb{E}\left[y^{4}(t)\right]}{\mathbb{E}^{2}\left[y^{2}(t)\right]}
$$

$$
g\left(\mathbb{E}\left[\Theta^{2}(t)\right]\right)=\Phi\left(\frac{\log \left(\frac{S}{K}\right)+r_{n} T-\frac{1}{2} \mathbb{E}\left[\Theta^{2}(t)\right]}{\sqrt{\mathbb{E}\left[\Theta^{2}(t)\right]}},,\right.
$$

is the kurtosis of the observed log-return $y(t)$,

and 


$$
\begin{aligned}
& f^{\prime \prime}\left(\mathbb{E}\left[\Theta^{2}(t)\right]\right)=\frac{1}{\sqrt{2 \pi}}\left(-\left(\frac{\mathbb{E}\left[\Theta^{2}(t)\right]-2\left(\log \frac{S}{K}+r_{n} T\right)}{4 \mathbb{E}\left[\Theta^{2}(t)\right] \sqrt{\mathbb{E}\left[\Theta^{2}(t)\right]}}\right)\left(\frac{\mathbb{E}^{2}\left[\Theta^{2}(t)\right]-4\left(\log \frac{S}{K}+r_{n} T\right)^{2}}{8 \mathbb{E}^{2}\left[\Theta^{2}(t)\right]}\right)\right. \\
& +\left(\frac{6\left(\log \frac{S}{K}+r_{n} T\right)-\mathbb{E}\left[\Theta^{2}(t)\right]}{8 \mathbb{E}^{2}\left[\Theta^{2}(t)\right] \sqrt{\mathbb{E}\left[\Theta^{2}(t)\right]}}\right) \exp \left\{-\frac{\left(2\left(\log \frac{S}{K}+r_{n} T\right)+\mathbb{E}\left[\Theta^{2}(t)\right]\right)^{2}}{8 \mathbb{E}\left[\Theta^{2}(t)\right]}\right\}, \\
& g^{\prime \prime}\left(\mathbb{E}\left[\Theta^{2}(t)\right]\right)=\frac{1}{\sqrt{2 \pi}}\left(\left(\frac{\mathbb{E}\left[\Theta^{2}(t)\right]+2\left(\log \frac{S}{K}+r_{n} T\right)}{4 \mathbb{E}\left[\Theta^{2}(t)\right] \sqrt{\mathbb{E}\left[\Theta^{2}(t)\right]}}\right)\left(\frac{\mathbb{E}^{2}\left[\Theta^{2}(t)\right]-4\left(\log \frac{S}{K}+r_{n} T\right)^{2}}{\mathbb{E}^{2}\left[\Theta^{2}(t)\right]}\right)\right. \\
& +\left(\frac{6\left(\log \frac{S}{K}+r_{n} T\right)+\mathbb{E}\left[\Theta^{2}(t)\right]}{8 \mathbb{E}^{2}\left[\Theta^{2}(t)\right] \sqrt{\mathbb{E}\left[\Theta^{2}(t)\right]}}\right) \exp \left\{-\frac{\left(2\left(\log \frac{S}{K}+r_{n} T\right)-\mathbb{E}\left[\Theta^{2}(t)\right]\right)^{2}}{8 \mathbb{E}\left[\Theta^{2}(t)\right]}\right\} .
\end{aligned}
$$

Proof of the theorem follows from Gong, Thavaneswaran and Singh [1] and Thavaneswaran and Singh [4]. Superiority of the approach has been demonstrated for Black-Scholes model with GARCH volatility and BlackScholes model with stochastic volatility in Gong, Thavaneswaran and Singh [1,2]. More details of related recent empirical results are also given in Cao and Guo [31,32].

\section{Conclusions}

Recently Gong, Thavaneswaran and Singh [1,2] have demonstrated the superiority of the truncated lognormal distribution method for option pricing by carrying out extensive empirical analysis of the European call option valuation for S \& P 100 index and showing that the proposed method outperforms other compelling stochastic volatility pricing models. In this paper, option pricing is discussed for Black-Scholes models, stochastic volatility models, pure jump process models and jump diffusion process models. Option pricing using PDE method together with FFT and the method based on a truncated lognormal distribution for Black-Scholes process and jump diffusion process are also discussed in some detail.

\section{References}

[1] H. Gong, A. Thavaneswaran and J. Singh, "A BlackScholes Model with GARCH Volatility,” The Mathematical Scientist, Vol. 35, No. 1, 2010, pp. 37-42.

[2] H. Gong, A. Thavaneswaran and J. Singh, "Stochastic
Volatility Models with Application in Option Pricing," Journal of Statistical Theory and Practice, Vol. 4, No. 4, 2010.

[3] E. Pillay and J. G. O’ Hara, "FFT Based Option Pricing under a Mean Reverting Process with Stochastic Volatility and Jumps," Journal of Computational and Applied Mathematics, Vol. 235, No. 12, 2011, pp. 3378-3384. doi:10.1016/j.cam.2010.10.024

[4] A. Thavaneswaran and J. Singh, "Option Pricing for Jump Diffusion Model with Random Volatility," The Journal of Risk Finance, Vol. 11, No. 5, 2010, pp. 496-507. doi:10.1108/15265941011092077

[5] F. Black and M. Scholes, "The Valuation of Option Contracts and a Test of Market Efficiency," Journal of Finance, Vol. 27, No. 2, 1972, pp. 99-417. doi:10.2307/2978484

[6] F. Black and M. Scholes, "The Pricing of Options and Corporate Liabilities,” Journal of Political Economy, Vol. 81, No. 3, 1973, pp. 637-659. doi:10.1086/260062

[7] R. C. Merton, “Theory of Rational Option Pricing,” Journal of Economics and Management Science, Vol. 4, No. 1, 1973, pp. 141-183.

[8] S. L. Heston, “A Closed-Form Solution for Options with Stochastic Volatility with Applications to Bond and Currency Options,” The Review of Financial Studies, Vol. 6, No. 2, 1992, pp. 327-343. doi:10.1093/rfs/6.2.327

[9] S. L. Heston, "Option Pricing with Infinitely Divisible Distributions,” Quantitative Finance, Vol. 4, No. 1, 2004, pp. 515-524. doi:10.1080/14697680400000035

[10] J. C. Cox, J. E. Ingersoll and S. A. Ross, “A Theory of the Term Structure of Interest Rates," Econometrica, Vol. 53, No. 2, 1985, pp. 385-407. doi:10.2307/1911242

[11] G. Bakshi, C. Cao and Z. Chen, "Empirical Performance 
of Altemative Option Pricing Models,” Journal of Finance, Vol. 52, No. 5, 1997, pp. 2003-2049.

doi: $10.2307 / 2329472$

[12] L. O. Scott, "Option Pricing When the Variance Changes Randomly: Theory, Estimation, and an Application,” Journal of Financial and Quantitative Analysis, Vol. 22, No. 4, 1987, pp. 419-438. doi:10.2307/2330793

[13] J. Hull and A. White, "The Pricing of Options on Assets with Stochastic Volatilities,” Journal of Finance, Vol. 42, No. 2, 1987, pp. 281-300. doi:10.2307/2328253

[14] J. Hull and A. White, "An Analysis of the Bias in Option Pricing Caused by a Stochastic Volatility,” Journal of International Economics, Vol. 24, No. 4, 1988, pp. 129145.

[15] P. Ritchken and R. Trevor, "Pricing Options under Generalized GARCH and Stochastic Volatility Processes,” Journal of Finance, Vol. 59, No. 1, 1999, pp. 377-402. doi:10.1111/0022-1082.00109

[16] J. B. Wiggins, "Option Values under Stochastic Volatilities,” Journal of Financial Economics, Vol. 19, 1987, pp. 351-372. doi:10.1016/0304-405X(87)90009-2

[17] S. L. Heston and S. Nandi, "A Closed-Form GARCH Option Valuation Model,” The Review of Financial Studies, Vol. 13, No. 1, 2000, pp. 585-625. doi:10.1093/rfs/13.3.585

[18] R. J. Elliot, T. K. Siu and L. Chan, "Option Pricing for GARCH Models with Markov Switching,” International Journal of Theoretical and Applied Finance, Vol. 9, No. 6, 2006, pp. 825-841. doi:10.1142/S0219024906003846

[19] P. Christoffersen, S. Heston and K. Jacobs, "Option Valuation with Conditional Skewness," Journal of Eco- nomtrics, Vol. 131, No. 1-2, 2006, pp. 253-284.

[20] L. Mercuri, "Option Pricing in a GARCH Model with Tempered Stable Innovations," Finance Research Letter, Vol. 5, No. 3, 2008, pp. 172-182. doi:10.1016/j.frl.2008.05.003

[21] A. M. Badescu and R. J. Kulperger, "GARCH Option Oricing: a Semiparametric Approach,” Insurance Mathematics and Economics, Vol. 43, No. 1, 2008, pp. 69-84. doi:10.1016/j.insmatheco.2007.09.011
[22] G. Barone-Adesi, H. Rasmussen and C. Ravanelli, "An Option Pricing Formula for the GARCH Diffusion Model," Computational Statistics and Data Analysis, Vol. 49, No. 2, 2005, pp. 287-310. doi:10.1016/j.csda.2004.05.014

[23] G. Barone-Adesi, R. F. Engle and L. Mancini, “A GARCH Option Pricing Model with Filtered Historical Simulation,” Review of Financial Studies, Vol. 21, No. 3, 2008, pp. 1223-1258. doi:10.1093/rfs/hhn031

[24] A. Thavaneswaran, S. Peiris and J. Singh, "Derivation of Kurtosis and Option Pricing Formulas for Popular Volatility Models with Applications in Finance,” Communications in Statistics.Theory and Methods, Vol. 37, 2008, pp. 1799-1814. doi:10.1080/03610920701826435

[25] A. Thavaneswaran, J. Singh and S. S. Appadoo, "Option Pricing for Some Stochastic Volatility Models,” Journal of Risk Finance, Vol. 7, No. 4, 2006, pp. 425-445. doi:10.1108/15265940610688982

[26] S. J. Taylor, “Asset Price Dynamics, Volatility, and Prediction,” Princeton University Press, Princeton, 2005.

[27] J. M. Steele, "Stochastic Calculus and Financial Applications,” Springer, New York, 2001.

[28] J. Vecer, "Stochastic Finance: A Numeraire Approach," Chapman \& Hall/CRC Press, London, 2011.

[29] H. Y. Wong and Y. W. Lo, "Option Pricing with Mean Reversion and Stochastic Volatility,” European Journal of Operational Research, Vol. 197, No. 1, 2009, pp. 179187. doi:10.1016/j.ejor.2008.05.014

[30] D. Duffie, J. Pan and K. Singleton, "Transform Analysis and Asset Pricing for Affine Jump-Diffusion,” Econometrica, Vol. 68, No. 6, 2000, pp. 1343-1376. doi:10.1111/1468-0262.00164

[31] L. Cao and Z. F. Guo, “Applying Gradient Estimation Technique to Estimate Gradients of European Call following Variance-Gamma," Proceedings of Global Conference on Business and Finance, Vol. 6, No. 2, 2011, pp. 12-18.

[32] L. Cao and Z. F. Guo, “Applying Variance Gamma Correlated to Estimate Optimal Portfolio of Variance Swap," Proceedings of International Conference of Financial Engineering, London, 6-8 July 2011. 